\title{
El Programa ULPN del Perú: Caracterización de las Prácticas de Enseñanza con Incorporación de Computadoras Portátiles
}

\author{
Carlos David Laura Quispe \\ Programa de Pos Graduación en Educación (PPGEDU) \\ Universidad Federal de Rio Grande (FURG) \\ Av. Italia Km 8, Carreiros, CEP: 96201-900, Rio Grande/RS, Brasil \\ cdavidlaura@gmail.com
}

\begin{abstract}
RESUMEN
El presente artículo se basa en algunos hallazgos de la investigación "Evaluación del impacto del programa OLPC sobre los procesos de mejoramiento de la educación pública", que tuvo como propósito central determinar si el programa "Una Laptop por Niño" (en adelante ULPN), incrementa el rendimiento académico de los alumnos beneficiarios del mismo. Desde una perspectiva cualitativa y a través de la realización de entrevistas semi estructuradas y observación no participante, se abordan las percepciones de maestros y maestras sobre la mediación de la computadora portátil en sus prácticas pedagógicas. Considerando los objetivos de la implementación del programa ULPN, se esperaría que la aplicación del mismo cambiaría la estructura y el método pedagógico de las prácticas escolares. No obstante $\mathrm{y}$, de acuerdo con los hallazgos encontrados, se pudo notar que los profesores no habían modificado la estructura ni el método pedagógico de sus clases por la incorporación de la computadora portátil. Hace seis años que los maestros y maestras vienen trabajando con las computadoras personales, sin embargo, no saben usarlas, ni mucho menos para que usarlas.
\end{abstract}

Palabras Clave: modelo pedagógico 1:1, percepción docente, actitudes, expectativas, discursos, cambio educativo.

\section{The ULPN Peru Program: Characterization of Practice Teaching to Incorporate Notebooks}

\begin{abstract}
This article is based on some research findings "Evaluating the impact of OLPC on the processes of improving public education," which had as its purpose to determine whether the "One Laptop per Child" (hereinafter ULPN) program increase the academic performance of students beneficiaries. From a qualitative perspective and through conducting semi -structured interviews and non- participant observation, the perceptions of teachers on mediation laptop in their teaching are discussed. Considering the objectives of the program implementation ULPN is expected to change the application of the same structure and pedagogical method of school practices. However, and according to the findings, it was noted that the teachers had not changed the structure and the teaching method of their classes by incorporating laptop. Six years ago the teachers have been working with personal computers, however, do not know to use them, much less to use.
\end{abstract}

Keywords: Pedagogical model 1:1, teacher perception, attitude, expectation, speeches, discourses, educational change. 


\section{INTRODUCCIÓN}

En las últimas décadas las tecnologías de la información y la comunicación (TIC) se han expandido a través de la sociedad y han tomado un rol protagónico dentro del hogar, la vida familiar, tanto a nivel sociodemográfico como en las dinámicas, estilo de vida, expectativas culturales y actitudes (Livingstone \& Helsper, 2007). En la actualidad la mayoría de gobiernos latinoamericanos están apostando por grandes proyectos educativos que involucran incorporación de tecnología portátil en las aulas. En especial los “modelos pedagógicos 1:1"-aprendizajes basados en una computadora por alumno-son los que están extendiéndose de modo creciente por toda la región. Por ejemplo, Um Computador por Aluno (UCA), de Brasil; Paraguay Educa, de Paraguay; Plan CEIBAL, de Uruguay; Conectar Igualdad, de la Argentina; Laboratorio Móvil Computacional (LMC), de Chile; Por la senda del 1:1, de Costa Rica; Educación y Cultura Digital, de México, Canaima de Venezuela, entre otros. Pero, ¿Por qué optar por los modelos pedagógicos 1:1? Algunas de las justificaciones para implementar modelos pedagógicos 1:1 que dan los que establecen las políticas incluyen el progreso educativo, social y económico, o una combinación de esos factores (CEPAL, 2008). Algunas iniciativas buscan mejorar la competitividad económica de sus países, preparando a los estudiantes para un mercado laboral saturado de tecnología. Otras se centran en la igualdad de acceso a los recursos digitales y la reducción de la brecha digital. Para otras iniciativas, el énfasis principal es mejorar la calidad de la educacióncomo el caso peruano-mediante prácticas nuevas tales como el aprendizaje centrado en el estudiante. Si bien estos tres énfasis no son contradictorios ni se excluyen unos a otros, su priorización es crucial para medir apropiadamente los impactos deseados (Valiente, 2011).

El Perú, siguiendo la tendencia internacional, se incorporó al programa ULPN en el año 2007. El mismo año, el Ministerio de Educación (MINEDU) a través de su Dirección General de Tecnologías Educativas (DIGETE) comenzó a entregar las computadoras portátiles - en la actualidad el Perú es el país que más computadoras portátiles adquirió en el mundo, alrededor de 980 mil computadoras-, capacitación para profesores y asistencia técnica. Sin embargo, es Uruguay el único país que ha conseguido implementar el proyecto bajo el “modelo pedagógico 1:1", es decir una computadora portátil para cada niño. Ni el Perú ni Uruguay, países con ingresos medios y con población rural inferior al 30\% del total, tienen el perfil de los países que OLPC habían pensado originalmente para la transformación de la educación con computadoras (Villanueva \& Olivera, 2012). El Programa ULPN, en su primera etapa, fue destinado a los estudiantes y docentes de las escuelas de Educación Primaria de las áreas rurales en extrema pobreza, con la finalidad de utilizarlas como herramientas pedagógicas, que permitan contribuir a lograr rápidamente la equidad educativa en pequeños poblados de la Costa, la Sierra y la Selva, donde tradicionalmente existe una enorme brecha digital con respecto a las áreas urbanas (MINEDU, 2008).

Junto con implementar la infraestructura tecnológica, el Ministerio de Educación se planteó la necesidad de capacitar a los docentes en el manejo de las computadoras portátiles, que estaba poniendo a su alcance en el espacio cotidiano de trabajo. Para la mayoría de los profesores este era el primer encuentro con ambientes de aprendizaje $1: 1$, por lo que la capacitación estuvo fuertemente centrada tanto en aspectos técnicos como pedagógicos (MINEDU, 2007). Sin embargo, las capacitaciones fueron relativamente cortas, se estipuló una capacitación de 20 horas pedagógicas, se confió en 
las capacidades pedagógicas de los profesores para que ellos desarrollaran la integración de las computadoras en los procesos de enseñanza y aprendizaje (Villanueva, 2007).

En ese contexto, diversos especialistas alertan sobre el real alcance de estas políticas, por ejemplo Warschauer y Ames critican la visión tecnocentrista que adopta el planteamiento de las iniciativas "One Laptop per Child” (OLPC), que se desarrollan en algunos países del tercer mundo afirmando que: "OLPC representa la última versión de una larga lista de planes de desarrollo tecnológico utópico que han intentado sin éxito resolver problemas sociales complejos con soluciones excesivamente simplistas" (Warschauer \& Ames, 2010, p. 33). De modo similar Villanueva (2011, p. 7) en relación a la implementación del programa ULPN en el Perú, señala:

[...] tenemos la herramienta (computadora personal), ahora cambiemos todo para acomodar la herramienta. Los pocos recursos que queden tras la compra de los aparatos serán usados para adaptar todo el sistema educacional para que funcione con la herramienta, desde software, medidas adecuadas de seguridad, entrenamiento y capacitación a profesores, estrategias metodológicas, sostenibilidad del programa, escalabilidad del mismo, etc. $Y$ sobre todo: las metas originales del sistema educacional serán cambiadas para acomodar a la herramienta.

Frente a este escenario, la literatura especializada muestra que cuando hay señales de efectos del uso de las computadoras portátiles en los aprendizajes, ello está vinculado no necesariamente al simple acceso o a un uso más intensivo sino a ciertos tipos de uso de las computadoras portátiles y también a las características del contexto del estudiante. El problema aquí, es que los análisis de este tipo de estudios no logran esclarecer de forma consistente cuáles son esos tipos de uso o las razones detrás de la relación positiva o negativa entre ciertos tipos de uso y resultados de aprendizaje (Condie \& Munro, 2007). Esto hace en cierto modo, que nuestro conocimiento sobre las características de las prácticas pedagógicas mediadas por la computadora portátil sea un poco difuso y vago.

En concreto, los estudios muestran que estas políticas de incorporar tecnología portátil al aula han resultado en un dramático incremento en el número de computadoras en colegios y salas de clase, y el acceso de los alumnos y profesores a Internet (Cristia et al., 2012). En esta misma línea, Selwyn (2004, p. 384), sostiene: “El acceso a la tecnología es inútil sin las habilidades, los conocimientos y apoyo para utilizarla de manera eficaz". Asimismo, Malamud \& Pop-Eleches (2011), sostienen que el uso intensivo de la computadora en programas como "One Laptop Per Child” en el hogar por parte de adolescentes, constituye una pérdida de tiempo para fines educacionales. Por otra parte, la investigación respecto a este tema es relativamente escaza, especialmente en países en vías de desarrollo, y como puede observarse, los estudios en el área han sido realizados principalmente en países desarrollados (Hinostroza et al., 2012). Por otra parte, el primer país en adoptar el Programa OLPC fue Uruguay, en una investigación exploratoria sobre el cambio de las prácticas docentes, debido a la incorporación de la computadora portátil se concluye que con las evidencias disponibles a partir de la investigación, no es posible afirmar que los docentes están cambiando sus prácticas como consecuencia del Plan Ceibal. Los autores señalan que no basta con instalar computadoras portátiles en las aulas, o como el plan Ceibal, incorporar computadoras portátiles con la modalidad 1:1. Además, es necesaria una profunda transformación del modelo docente así como un cambio en el nivel de gestión institucional de las escuelas (Fernández et al., 2009). 
Por tanto, se asume que la incorporación efectiva de la computadora portátil en el aula es un proceso complejo (Zhao et al., 2001) y necesita de más análisis para lograr una mayor comprensión del fenómeno. Esta situación, motivó el interés por desarrollar un estudio que pudiera investigar las características de las prácticas pedagógicas mediadas por la computadora portátil. Es de vital importancia contar con metodologías que permitan la evaluación sistemática del Programa ULPN, para que sus resultados sirvan de base tanto para el mejoramiento de dichas experiencias, como para la orientación, diseño y planificación de nuevos proyectos. En ese sentido, se espera que éste estudio constituya un complemento, a la discusión objetiva y productiva en torno a las mejores posibilidades de incorporación de las computadoras portátiles, en escuelas de educación básica del Perú.

\section{ASPECTOS METODOLÓGICOS}

Este estudio buscó conocer y describir las prácticas pedagógicas con incorporación de las computadoras portátiles. Así, los objetivos específicos de esta investigación son: i) identificar la organización de las prácticas pedagógicas con la incorporación de la computadora portátil. ii) describir la dinámica de las prácticas pedagógicas con la incorporación de las computadoras portátiles. A través de esta investigación se buscó responder a las siguientes preguntas: ¿Cuáles son las características que poseen las prácticas pedagógicas con la incorporación de computadoras portátiles?, ¿Qué rol juega la computadora portátil en las prácticas pedagógicas escolares? Para responder estas preguntas, se asume que de haber una modificación en la estructura y el método pedagógico de las prácticas pedagógicas, estas deberían reflejar un cambio de actitud de los profesores.

El universo del estudio estuvo constituido por maestros y maestras de escuelas públicas de la región Arequipa, Perú. La muestra estuvo constituida por 36 escuelas, sin pretensión de representatividad estadística. Se seleccionó al director (en escuelas polidocentes) y a un docente por institución educativa que pueda brindar la mayor información posible sobre la caracterización y roles de la computadora portátil en las aulas. En total fueron entrevistados y observados 87 maestros y maestras. El proceso de recolección de datos incluyó dos instrumentos: entrevista semiestructurada y pauta de observación de prácticas de aula con las computadoras personales.

El análisis de la información levantada se plantea como parte de un proceso global y se constituye como una pieza clave para la búsqueda de las respuestas a las interrogantes de la investigación. De esta manera, los procedimientos de análisis tienen su origen en el planteamiento de las preguntas de investigación, que permitieron definir las categorías con las cuales se analizan las entrevistas y observaciones. Las entrevistas fueron grabadas en audio y, luego se transcribió para realizar la respectiva codificación utilizando la herramienta tecnológica QSR NVIVO 7.

\section{PRINCIPALES HALLAZGOS}

Los resultados se presentan considerando las perspectivas de los distintos actores en la investigación: directores y profesores pertenecientes a las áreas fundamentales del currículo. Todos los datos fueron obtenidos a través de las entrevistas realizadas a los directores y docentes lo que es complementado con los datos recopilados mediante la observación. La información ha sido estructurada considerando las dimensiones que guiaron la indagación. Por esto, es la sección correspondiente a la mirada de los 
profesores sobre el programa ULPN, se presentan las opiniones y percepciones de los docentes según estas dimensiones: caracterización de los ambientes de aprendizaje mediados por la computadora portátil y, rol que cumplen las computadoras personales. Describimos los resultados generados, mediante un enunciado descriptivo y, en algunos casos, citas recogidas directamente de los textos de los sujetos informantes (directores y profesores). Es preciso destacar, que con el propósito de proteger el anonimato de informantes e instituciones que participaron del estudio, a cada uno de los informantes se les asignó un código (por ejemplo: Profesor C007).

\subsection{Características de las clases con incorporación de computadoras portátiles}

Los profesores son los actores directos cuando, de incorporación tecnológica en el aula se trata. El profesor es naturalmente la primera persona que puede observar los factores que afectan el uso de la tecnología en el aula. Son por lo tanto, los principales observadores, ejecutores y evaluadores, lo cual no quiere decir que sean los que realicen estas tareas de la mejor manera (Zhao et al., 2001). El papel del profesor es decisivo para que se lleve a cabo un uso efectivo y eficiente de las computadoras portátiles. El uso de los recursos tecnológicos en el aula sólo podrá servir como instrumento de aprendizaje si los profesores saben usarlo y si consideran que complementa su labor pedagógica, si estos tienen claro que es lo van hacer con la tecnología. Esto significa que es necesario tener en cuenta que para poder utilizar las computadoras personales en forma efectiva, el profesor requiere conocer las potencialidades y limitaciones de estas y, sobre todo su aplicación concreta y correcta en el aula (Trahtemberg, 2010).

En efecto, la apropiación de la computadora portátil por parte de los profesores demanda nuevos e importantes papeles a los profesores, y la necesidad de que éstos conozcan y dominen tales tecnologías (Rivero, 1999). Un punto fundamental es que los profesores deberán redefinir sus roles tradicionales, aprender, codificar y dosificar las tareas programadas en base a las computadoras portátiles, y para esto deberían conocer a profundidad tanto los aspectos técnicos así como nuevas metodologías de enseñanza y aprendizaje, basadas en la mediación de la computadora portátil (Zucker, 2005). Pero, para lograrlo, no es suficiente ofrecer a los profesores cursos y capacitaciones esporádicas como se ha venido haciendo. Es preciso formar al profesor, cambiar su visión sobre las nuevas tecnologías, preparándolo para trabajar correctamente con ellas, para producir constantemente más y mejores materiales didácticos, siempre en equipo, para usar de forma adecuada estos productos de inteligencia artificial (Siqueira, 2004).

El programa ULPN tiende a cambiar la organización de la escuela: distribución de espacios, horarios, práctica docente y funciones de los profesores. El uso de las computadoras portátiles implica cambios en las actitudes de los profesores que permitan innovar en los procesos de enseñanza y aprendizaje. Estos cambios obligan a romper con las prácticas tradicionales en las cuales prevalece la clase frontal y el concepto del profesor como un ente rígido. Es decir, los profesores deberían modificar la manera de cómo enseñan a sus estudiantes, lo que puede incluir cambiar el rol que desempeñan en la sala de clases y la forma en la que sus aulas están organizadas físicamente. Sin embargo, se encontró que la mayoría de profesores ponía fuerte resistencia a tales cambios, como se puede percibir en el siguiente fragmento: Nosotros nos formamos en otra época, se puede decir que tanto nuestra formación primaria, secundaria y formación como docentes fue netamente tradicional [...] pero nuestros alumnos nacieron con esto, para ellos no existe complicación alguna, nosotros tratamos de 
aprovechar la computadora, pero no es fácil, es bastante complicado [...] mire, en mi caso vienen las niñas del pedagógico y me preparan todo lo que voy a utilizar en el desarrollo de mi clase, sólo así puedo hacer uso de la computadora [...] para serle franca, yo no podría usar las computadoras sin la ayuda de las practicantes del instituto (Profesor C001).

Otro aspecto a destacar, es que el docente pone en evidencia su temor a la tecnología, su condición de no nativo de la tecnología y su resistencia al cambio. Su condición de no nativo de la tecnología le hace sentirse en desventaja frente a sus alumnos, siente que pierde el protagonismo en el aula y por lo tanto, incapaz de conducir el proceso pedagógico. Por lo tanto, el profesorado siente una falta de confianza para la utilización de las computadoras portátiles, y como consecuencia si el profesorado no confía en su competencia para manejar las computadoras esto supone un serio obstáculo en su voluntad para apropiarse de éstas en el aula (BECTA, 2004). Lo anterior se sustenta en la siguiente declaración: Los papas les compramos computadora a los hijos y en un cinco nos dan clases [...] mis alumnos descubren los recursos de la computadora muy rápidamente. Que el alumno sepa más que yo, es una gran desventaja porque yo no podría conducir un proceso si no estoy a la cabeza [...] yo pienso eso, mis alumnos me pueden engañar fácilmente en el uso de la computadora [...] yo lo he probado con mis hijos, sobrinos y nietos le dan duro a la computadora y uno tiene miedo, temor echar a perder la computadora y el niño no, él mete los dedos y hace lo que quiere (Profesor J014).

No obstante, para las nuevas generaciones, llamados por algunos como "seres digitales" las tecnologías son una herramienta más, para ellos no son importantes las instrucciones, ellos primero comienzan a jugar, en caso de encontrar problemas, llaman a un amigo, buscan información en internet o envían un mensaje para un fórum (Veen \& Vrakking, 2009). En relación a lo anterior un profesor expone lo siguiente: nosotros muchas veces actuamos de manera esquemática, sin embargo los niños van buscando nuevas cosas, es más como le decía hace unos momentos hay cosas que no nos había enseñado, incluso por el corto tiempo que nos capacitaron, hay muchas cosas que quedaron inconclusas, entonces los niños salían miren he descubierto esta nueva actividad, miren profesora aquí había sobre geografía, y ellos nos van enseñando, en muchas ocasiones he encontrado a alumnos que saben más que yo respecto al manejo de estos equipos, ellos han comenzado a traer en sus USB algunas cosas, música, entre otras cosas, que jalaban de internet bueno, esto lo vaciaban a la computadora (Profesor I003)

Por otro lado, un gran número de docentes informantes no están plenamente convencidos, que el uso de la computadora portátil ayude a mejorar los procesos de enseñanza y aprendizaje, y por ende haya mejores resultados académicos en los alumnos. Al parecer los docentes no esperan grandes cambios en su labor docente, como consecuencia de la incorporación de la computadora portátil. Esto coincide con lo que plantea la literatura especializada al respecto, en relación a que la presencia de la tecnología no es tan fuerte como para modificar las creencias y convicciones de los profesores en relación a cómo enseñar (Goodison, 2003; Zucker \& Bonifaz, 2005). La profesora A008 menciona: pienso que si la computadora no es bien utilizada, en vez de ayudarnos al proceso educativo [...] perjudica te lo digo categóricamente, aquí en la escuela tenemos las computadoras cerca de cuatro años [...] pero aún no podemos identificar los resultados (Profesor A008). 
Finalmente, en las observaciones realizadas se pudo notar que los profesores no habían modificado la estructura ni el método pedagógico de sus clases por la inclusión de las computadoras portátiles, más aún, fue posible observar que estos recursos habían sido incorporados a una metodología que sin duda era considerada como adecuada por los propios profesores. En general, los profesores tienden a confiar más en lo que tradicionalmente les ha funcionado, por lo cual no están abiertos a innovaciones que involucren computadoras. Bajo este supuesto los profesores estarían en la etapa de adaptación de la tecnología, en la cual los profesores ya han incorporado las tecnologías como recurso en sus clases, pero manteniendo sus prácticas pedagógicas tradicionales, es muy probable, entonces que si no se hubieran utilizado las computadoras, el tipo de actividad final habría sido muy similar pero con otros recursos (Sandholdz, Ringstaff \& Dwyer, 1997).

\subsection{Roles de la computadora portátil en las prácticas pedagógicas escolares}

La implementación de un programa de innovación tecnológica en el aula, es un proceso complejo que toma su tiempo y depende de una serie de factores que deben ser considerados para lograr el éxito (Kosma \& McGhee, 2003). Probablemente, una de las mayores limitaciones es que el Programa adolece, en su ejecución de problemas pedagógicos. En la actualidad, frente al proceso de incorporación de las computadoras portátiles en el Perú, podemos encontrar posiciones extremas que van desde la utópicas hasta las escéptica. Las primeras perciben a las computadoras portátiles como el remedio a los problemas de la educación en el País, por ejemplo Becerra (2010), afirma, lo maravilloso de las computadoras portátiles, es que están diseñadas como una herramienta de aprendizaje, es que se puede, desde una misma plataforma, elevar la calidad de la educación sin presiones, no hay un plan que demande un número de horas de trabajo con ellas, ya que se basa en el uso libre para estimular la creatividad. Esta forma de pensar es común en el Perú, y plantea que la realización personal es la solución a una serie de males sociales, incluida la mala educación. Y las segundas, consideran que si no hay un diseño instruccional que garantice que las computadoras sean incorporadas en el currículo y la práctica en el aula orgánicamente, y no solo como maravillas emocionalmente poderosas; si no se cuenta con las fuentes de información adecuadas y pertinentes al plan de estudios; si los profesores no tienen mayor idea de cómo usarlas, y son fácilmente superados por los alumnos; y finalmente: Si la computadora conectada a Internet irrumpe en el aula sin considerar los problemas que crea tanto como las ventajas que puede generar, no hay ninguna razón para pensar que va a mejorar la educación. La va a cambiar, pero no necesariamente para bien (Villanueva \& Olivera, 2012).

Una vez puesto en marcha el programa ULPN en el Perú, se da inicio a una serie de creencias negativas sobre este. En la realidad estudiada, la mayoría de los informantes coinciden en que desde las instancias del Ministerio de Educación los objetivos a alcanzar no estuvieron claros desde el inicio. Por ejemplo, el profesor I011 sostiene: En ningún momento se especificó para qué [...] de pronto se dijo el Perú entra al programa una laptop por niño, qué bueno que todas las escuelas del Perú tengan computadoras, que el estado invierta en esto, entonces, que bien estamos a la vanguardia en tecnología, tenemos tecnología pero, qué haces con la tecnología, nunca se nos dijo para qué era [...] es que nunca me dijeron cuando esto comenzó, para qué quería yo las computadoras [...] que quiero con eso (Profesor I011). 
En el momento actual, el programa ULPN tiende a desajustar las rígidas estructuras de la escuela: En cuanto a distribución de espacios, horarios, funciones de los profesores, etc. El profesor siente que su ambiente de trabajo ha sido perturbado, de allí que tenga una actitud de rechazo o desconfianza sobre su efectividad. Esta actitud posiblemente le lleve a ver cierto tipo de riesgos en su uso, le impida determinar el rol de la computadora portátil y no vea las ventajas pedagógicas que le ofrece. Al respecto, los profesores afirman: Qué tienes que cumplir con las programaciones, que tienes que cumplir con evaluaciones, que hay que tener las planificaciones para tal día, o sea yo creo es una cuestión de nuestro sistema escolar [...] recalco no me refiero al tiempo que tú usas en el aula, sino que al tiempo que necesitas para planificar las actividades; para buscar, para crear, para indagar, para probar el material, a veces no en el salón de clases. En el salón de clases tienes que presentar algo que funcione, ¿verdad? Pero la falta de tiempo en tu casa, en tu computadora, yo creo que eso desmotiva trabajar con las computadoras (Profesor N009).

En lo que respecta a los roles en sí, la mayor parte de estos tienen como finalidad la motivación o presentación de nuevos contenidos. Al parecer el programa ULPN, no ha propuesto una estrategia que modifique las prácticas pedagógicas en el aula; solo ha propuesto un cambio en cuanto a la cantidad de información disponible. En relación a los momentos en que usan las computadoras XO, los profesores ubicaron el uso de las computadoras portátiles siempre al iniciar o finalizar la sesión pedagógica. Al respecto el director C004 dice: en realidad el uso que le dan los profesores en esta escuela es muy limitado, y algunos profesores simplemente no la usan, si no estuvieran las computadoras, es probable que todos los profesores estarían reclamando que no tenemos computadoras que no tenemos esto, no tenemos materiales [...] osea recursos diría yo como al máximo, sin embargo el profesor no está comprometido, yo ahí hago una crítica fuerte al profesor con su propio desarrollo, ósea porque tengo que esperar que el director me ordene que yo utilice la computadora, si hoy en día en todas partes se usa, todo el mundo lo usa, y mis alumnos van a tener ese requerimiento cuando salgan a la vida del trabajo (Director C004).

En síntesis, estas máquinas no han alcanzado una calidad de fabricación de "nivel productivo", los programas no han funcionado como se prometió en un primer momento. En líneas generales, se puede afirmar que el programa OLPC no ha tenido el éxito de conseguir una computadora que funcione como se deseaba, ni el de convencer a la suficiente cantidad de gobiernos, organismos caritativos o filántropos para comprarlas y distribuirlas (Derndorfer, 2010).

\section{REFLEXIONES FINALES}

A partir de la investigación realizada, el análisis de los resultados encontrados y lo que nos indica el estado del arte es posible señalar las siguientes conclusiones:

La implementación de este programa en el Perú, llamado ULPN, no ha estado ajeno a dificultades propias de una innovación que genera cambios fuertes en un espacio que ha sido muy estable: La sala de clases. En general, el principal problema fue ponerlo en práctica sin haber realizado un exhaustivo diagnóstico, ponderando los costos y beneficios que generaría, considerando los pros y contras para su aplicación y las condiciones previas que se requieren para su aplicación a la realidad peruana. 
Más aun, durante la implementación y desarrollo del programa, se ha encontrado dificultades que se relacionan fuertemente con los profesores, desconocimiento de metodologías o estrategias de trabajo que permitan una adecuada utilización de las computadoras, desconocimiento en cuanto al manejo y resolución de aspectos técnicos al utilizar las computadoras portátiles, falta de confianza en el uso de estas nuevas tecnologías en la enseñanza. El temor a las tecnologías, sus creencias negativas, su condición de no nativo de la tecnología y su rechazo al cambio de actitud, constituyen limitaciones docentes preponderantes para la integración de la computadora al aula. En síntesis, los profesores aún no generan grandes cambios a nivel pedagógico y tampoco saben cómo realizar un proceso de reflexión sobre su desempeño utilizando las computadoras portátiles.

No es fácil lograr modificar características tan personales como las anteriormente descritas, posiblemente en muchos casos no se llegue nunca a influir en ellas, pero si es posible independizar la implementación de dichas características, mediante una mejor estructuración de la integración que se espera, con políticas internas que la potencien, por otra parte, si se logran manejar adecuadamente las demás variables referidas a mejor preparación docente, infraestructura suficiente y adecuada, soporte técnico y pedagógico, es posible que la influencia que tienen las características personales de los profesores, no proclives a la integración, no sea una limitación si no una oportunidad. Parece ser que los profesores más innovadores, que abren las puertas a nuevas tecnologías al interior del aula y que buscan constantemente nuevas formas de realizar el proceso de enseñanza y aprendizaje, se inclinen más a la integración de la tecnología en sus clases, que los profesores más tradicionales.

Aún no existe suficiente evidencia que sustente los aprendizajes 1:1 en el contexto rural Peruano, con sus bondades y falencias, pero el proyecto ULPN nos enfrenta a una realidad que ha sido implementada en otras latitudes y que han mostrado que en una implementación cuidadosa, que contemple las necesidades de los profesores y los requerimientos del contexto puede apoyar enormemente el mejoramiento de la calidad y equidad de la educación básica rural. Sin embargo es necesario promover políticas a nivel institucional, infraestructural y en relación con la preparación de los profesores en los siguientes aspectos: capacitar a los profesores en aspectos pedagógicos referidos a la integración del portátil en la enseñanza y aprendizaje; perfeccionar a los profesores en el manejo tecnológico del portátil y los recursos incluidos, incluyendo prepararlos para resolver problemas técnicos; establecer una política de integración curricular del portátil, teniendo en cuenta lo que deben aprender los alumnos y las metodologías más apropiadas en donde se integre el uso de la computadora y las prácticas pedagógicas que lo acompañan, como en los cambios metodológicos que estos introducen; estructurar nuevos módulos de aprendizaje relacionados con el contexto en donde se ubica la escuela. El programa ULPN exige, por lo tanto, un nuevo perfil del profesor, el cual debe considerar no sólo actualización y perfeccionamiento sino también el de competencias tecnológicas, las que son necesarias incluir en las políticas de nuevas contrataciones y nombramientos de profesores.

Agradecimiento: al Consorcio de Investigación Económica y Social (CIES). De Lima Perú. Por el financiamiento para la realización del presente estudio. Proyecto PMP01.

\section{REFERENCIAS}


BECERRA, O. ¿What is reasonable to expect from information and comunications technologies in education? (¿Qué es razonable esperar de las tecnologías informáticas y comunicativas en la educación) Educational technology debate. Agosto, 2010. Disponible en: <https://edutechdebate.org/computerconfigurationsfor-leraning/what-is-reasonable-toexpect-from-information-and comunicationtechnologies-in-education>. Acceso en: 18 de enero de 2012.

BRITISH EDUCATIONAL COMMUNICATIONS AND TECHNOLOGY AGENCY (BECTA). Corporate Plan 2004-2007. Coventry, UK. 2004.

CEPAL. Las políticas de tecnología para escuelas en América Latina y el mundo: visiones y lecciones. Santigo. 2008.

CONDIE, R.; MUNRO, B. The Impact of ICT in Schools: a landscape review. UK: Becta. 2007.

CRISTIA, J.; IBARRARÁN, P.; CUETO, S.; SANTIAGO, A.; SEVERIN, E. Technology and Child Development: Evidence from the one laptop. IDB

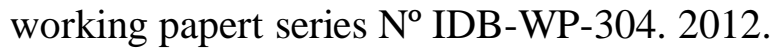

DERNDORFER, C. OLPC in Perú: A problematic Una Laptop Por Niño program. Educational technology debate, Setiembre, 2010. Disponible en: $<$ http://edutechdebate.org/olpc-in-south-america/olpc-in-peruone-laptop-perchild-problems >. Acceso en: 10 de Noviembre de 2012.

FERNÁNDEZ, F.; BOCHIA, F.; DURÁN, R.; RODRÍGUEZ, Z. Estudio exploratorio sobre la percepción del impacto del Plan Ceibal: ¿Cambian las prácticas de los docentes? Montevideo. 2009.

GOODISON, T. Integration ICT in the classroom: a case study of two contrasting lesson. 2003.

HINOSTROZA, E.; IBIETA, A.; LABBÉ, C.; ISAACS, M. Estudio exploratorio de la relación entre las percepciones y usos de computadores e internet de apoderados y alumnus de enseñanza media. Instituto de Informática Educativa. Universidad de la Frontera. 2012.

KOZMA, R., McGHEE, R. ICT and innovative classroom practices, in R.B. kozma (Ed.), Technology, Innovation and Educational Change (pp. 43-80). Eugene: International Society for Technology in Education. 2003.

LIVINGSTONE, S.; HELSPER, E. Gradations in digital inclusion: children, young people and the digital divide. New Media \& Society, 9(4), 671-696. 2007.

MALAMUD, O.; POP-ELECHES, C. Home Computer Use and the Development Human Capital. Quarterly Journal of Economics. Vol. 126, No 2: pp. 987-1027. 2011.

MINEDU. El Programa una Laptop por Niño. Construyendo un futuro mejor para los peruanos. Lima. 2007.

MINEDU. Manual del docente para el uso de la laptop XO. Dirección General de Tecnologías Educativas. Lima. 2008.

RIVERO, J. Educación y exclusión en América Latina. Reformas en tiempos de globalización. 2da edición. Lima: Tarea. 1999.

SANDHOLTZ, J.H., RINGSTAFF, C. y DWYER , D.C. Teaching with Technology: Creating Student-Centered Classrooms. New York: Teachers College Press. 1997.

SELWYN, N. Reconsidering political and popular understandings of the digital divide. New Media \& Society, 6(3), pp. 341-362. 2004.

SIQUEIRA, E. 2015-Como viviremos. Sao Paolo. Brasil: Ed. Saraiva. 2004. 
TRAHTEMBER, S. El impacto previsible de las nuevas tecnologías en la enseñanza y organización escolar. Seminario internacional "el futuro de la educación en América Latina y el Caribe”. 2010.

VALIENTE, O. Los modelos 1:1 en educación. Prácticas internacionales, evidencia comparada e implicaciones políticas. 2011.

VEEN \& VRAKKING. Homo Zappiens. Educando na era digital. Artmed. Editora. São Paulo. 2009.

VILLANUEVA, E. Sobre OLPC en el Perú. Departamento de Comunicaciones. Pontificia Universidad Católica del Perú. 2007. . Sobre OLPC en el Perú. Departamento de Comunicaciones. Pontificia Universidad Católica del Perú. 2011.

VILLANUEVA, E.; OLIVERA, P. Barreras institucionales para el desarrollo de una innovación: evaluando la implementación de las computadoras $\mathrm{XO}-1$ en dos escuelas periurbanas del Perú. Investigación sobre TIC en Latinoamérica. Volumen 8, N 4. 2012.

WARSCHAUER, M.; MORGAN, A. ¿Can one laptop per child save the world's poor?. Journal of international affairs, fall/Winter. Vol. 64. No 1. 2010.

ZHAO, Y., PUGH, K., SHELDON, S., BYERS, J.L. Conditions for Classroom Technology Innovations. Teachers College Record. Vol. 104 Nº 3, April 2002, pp. 482-515. 2002.

ZUCKER, A.; BONIFAZ, A. Lessons Learned About Providing Laptops For All Students. NEIR-TEC. 2005. 